\title{
3 Research Square

\section{An improved deyolking procedure facilitates the proteomics analysis of early developmental stages of zebrafish embryos}

\section{Purushothaman Kathiresan}

Nord University

Christopher Presslauer

Nord University

Prem Prakash Das

National University of Singapore

Lim Teck Kwang

National University of Singapore

Steinar Dae Johansen

Nord University

Qingsong Lin

National University of Singapore

Igor Babiak ( $\square$ igor.s.babiak@nord.no)

Nord University https://orcid.org/0000-0002-1462-2690

Research article

Keywords: egg yolk, embryonic development, LC MS/MS shotgun proteomics, proteome, zebrafish

Posted Date: August 23rd, 2019

DOI: https://doi.org/10.21203/rs.2.13414/v1

License: (c) (i) This work is licensed under a Creative Commons Attribution 4.0 International License.

Read Full License 


\section{Abstract}

Background: Zebrafish is a well-recognised organism for investigating vertebrate development and human diseases. However, the data on zebrafish proteome are scarce, particularly during embryogenesis. This is mostly due to the overwhelming abundance of egg yolk proteins, which tend to mask the detectable presence of less abundant proteins. Results: In this study, we developed an efficient procedure to reduce the amount of yolk in zebrafish early embryos to improve the LC-MS-based shotgun proteomics analysis. We demonstrated that the deyolking procedure resulted in a greater number of proteins being identified. This protocol resulted in approximately two-fold increase in the number of proteins identified in deyolked samples at cleavage stages, and the number of identified proteins increased greatly by 3-4 times compared to non-deyolked samples in both oblong and bud stages. Gene Ontology and KEGG analysis revealed a high number of functional proteins differentially accumulated in the deyolked versus non-deyolked samples. The most prominent enrichments after the deyolking procedure included processes, functions and components related to cellular organization, cell cycle, control of replication and translation, and mitochondrial functions. Conclusion: This deyolking procedure improves both qualitative and quantitative proteome analyses and provides an innovative tool in molecular embryogenesis of polylecithal animals, such as fish, amphibians, reptiles, or birds.

\section{Background}

Zebrafish have become a prominent and broadly used model system to study developmental biology, neurogenetic disorders, genetics, toxicology, reproduction, pathology, and pharmacology [1-5]. The genome annotation is relatively well developed [6], and the embryonic transcriptome of zebrafish has been characterized in several studies [7-11]. However, knowledge about the comprehensive proteome dynamics during embryogenesis in zebrafish remains elusive.

Proteome in zebrafish is usually investigated in adult organs or tissues [12-15]. The overwhelming occurrence of vitellogenins - yolk proteins - is a limiting factor in a polylecithal embryo, such as in zebrafish, as it hinders global identification of less abundant proteins using mass spectrometry-based techniques $[4,16]$. Proteolytic peptides of yolk proteins can potentially subdue the ionization of the less abundant proteolytic peptides of non-yolk proteins $[17,18]$. Consequently, abundant yolk proteins can potentially interfere with the identification of cellular proteins, although the degree of such the interference is unknown. To reduce the abundance of yolk proteins, deyolking protocols are employed; they have been used in a number of studies on zebrafish embryos and larvae from 3.3 hours postfertilization (hpf) to 7 days post-fertilization (dpf) [16, 19-21]. In most extensive studies to date, 5267 and 8363 proteins were identified in zebrafish deyolked embryos at $24 \mathrm{hpf}[22,23]$.

So far, all the studies on zebrafish embryonic proteome were conducted on embryos being at a certain developmental advancement, and the information on the early stages, particularly before the maternal-tozygotic transition (MZT), is missing. Pre-MZT stages of development are characterized by rapid, synchronous cell cycles (cleavages), and the development is driven by maternally-provided factors, 
including transcriptome and proteome [24]. Therefore, a knowledge of maternal proteome dynamics seems to be essential for understanding the regulation of early embryonic development in zebrafish. We have improved the deyolking procedure, allowing the efficient capture and identification of proteins from the onset of development (1-cell stage). The protocol yielded 2 times more identified proteins compared to the non-deyolked counterparts in cleavage stages, and 3 to 4 times at oblong and bud stages. Also, the protocol caused minimal loss of proteins. Our improved protocol has been effective for the subsequent systematic proteomics studies of zebrafish early embryonic development, and it is applicable to studies on other polylecithal animals.

\section{Methods}

Fish

The samples were collected at the zebrafish facility of the Nord University, Bodo, Norway. The experimental process and husbandry were performed in agreement with the Norwegian Regulation on Animal Experimentation (The Norwegian Animal Protection Act, No. 73 of 20 December 1974). This was certified by the National Animal Research Authority, Norway, General License for Fish Maintenance and Breeding no. 17.

The maintenance of zebrafish was done using an Aquatic Habitats recirculating system (Pentair, Apopka, FL, USA) and following established protocols [31]. The fish were fed newly hatched Artemia sp. nauplii (Pentair) and SDS zebrafish-specific diet (Special Diet Services, Essex, UK) according to the manufacturers' instruction. The zebrafish used in the experiment were from the $A B$ line.

\section{Sample collection}

Embryos originated from natural spawning and were collected at five developmental stages (Fig. 4). Embryo development was monitored and staged according to Kimmel et al. [32]. For each developmental stage, embryo batches were divided into two variants: non-deyolked and deyolked. The non-deyolked (intact) embryos were promptly snap-frozen in liquid nitrogen and subsequently stored at $-80^{\circ} \mathrm{C}$. The deyolked embryo variants went through the process of dechorionation (removal of chorion) and deyolking. Additionally, the 1-cell ( $0.5 \mathrm{hpf}$ ) and high-stage (3.3 hpf) embryos were collected to compare our deyolking protocol with that by [16].

\section{Dechorionation and deyolking}


Embryos were placed in a Petri dish in phosphate-buffered saline (PBS) supplemented with $1.0 \mathrm{mg} / \mathrm{mL}$ Pronase (Sigma Aldrich, St. Louis, MO, USA) [31]. The enzymatic digestion of chorion was performed for 5 min at $37^{\circ} \mathrm{C}$ with gentle shaking. Embryos were washed minimum 5 times with PBS or until all visible chorion fragments were removed.

The dechorionated embryos were processed using our modified protocol with three-step deyolking and a single wash. The embryos were transferred to $1.5 \mathrm{~mL}$ Eppendorf tubes containing $1.0 \mathrm{~mL}$ of deyolking buffer ( $55 \mathrm{mM} \mathrm{NaCl}, 3.6 \mathrm{mM} \mathrm{KCl}$, and $1.25 \mathrm{mM} \mathrm{NaHCO}_{3}$ ) and were mechanically disrupted by pipetting repeatedly through a $100 \mu \mathrm{L}$ tip. The content was gently mixed by inverting the tube several times before centrifugation at 13,000 RPM for $1 \mathrm{~min}$ at $4^{\circ} \mathrm{C}$. The supernatant containing the yolk was discarded, and the pellet was re-suspended with the deyolking buffer, vortexed, and centrifuged as above. The procedure was repeated two times. After this, the pellet was re-suspended with $10 \mathrm{mM}$ Tris- $\mathrm{HCl}(\mathrm{pH} \mathrm{7.4),} \mathrm{vortexed,}$ and centrifuged as above. The supernatant was discarded and the pellet (deyolked embryos) was snapfrozen in liquid nitrogen and stored at $-80^{\circ} \mathrm{C}$. Additionally, for comparison of our protocol with that of [16], the dechorionated embryos at 1 cell and high stage were subjected to two types of deyolking protocols reported by [16]: (1) one-step deyolking, and (2) one-step deyolking with two additional wash steps.

\section{Protein extraction}

Both intact (non-deyolked) and deyolked embryo samples were lysed by adding $100 \mu \mathrm{L}$ of sodium dodecyl sulphate (SDS) lysis buffer (1 \% SDS; Sigma-Aldrich, St. Louis, MO, USA), 0.5 M triethylammonium bicarbonate buffer pH 8.5 (TEAB; Sigma Aldrich) and $1 \times$ Protease Inhibitor cocktail (Thermo Scientific, Rockford, IL, USA)). The tubes were vortexed and incubated at $90^{\circ} \mathrm{C}$ for $30 \mathrm{~min}$, then cooled on crushed ice for $5 \mathrm{~min}$. The lysed material was centrifuged at 13,000 RPM for 20 min at $4{ }^{\circ} \mathrm{C}$. The supernatant, containing the proteins, was collected and transferred to a new Eppendorf tube. The total protein concentration was quantified using a Qubit® 3.0 Fluorometer (Invitrogen, Eugene, OR, USA) and the Qubit ${ }^{\text {TM }}$ Protein Assay Kit (Invitrogen) according to the manufacturer's instructions. After the quantification, the samples were freeze-dried (VirTis BenchTop ${ }^{\mathrm{TM}} \mathrm{K}$, Warminster, USA) at $-80^{\circ} \mathrm{C}$ for $18 \mathrm{~h}$ before being shipped to the Department of Biological Sciences, National University of Singapore for proteomics analysis.

\section{Polyacrylamide gel electrophoresis}

One-dimensional gel electrophoresis was performed to check the efficiency of deyolking protocol, as well as to compare the efficiency of our protocol with the previous ones. Approximately equal concentrations 
of proteins from each sample were supplemented with $2 \times$ SDS loading dye. The samples were denatured by incubation at $95^{\circ} \mathrm{C}$ for $10 \mathrm{~min}$ and then the proteins were separated by SDS gel electrophoresis (4$20 \%$ Mini-PROTEAN® TGX ${ }^{\text {TM }}$ Precast Protein Gels, Bio-Rad, Hercules, California, USA) in SDS running buffer for $1 \mathrm{~h}$. Afterwards, the gel was washed with deionised water for $10 \mathrm{~min}$. The gel was stained with Coomassie Blue (Coomassie Brilliant Blue R-250, Bio-Rad) for 20 min, and de-stained with de-staining solution ( $40 \%$ methanol $+10 \%$ acetic acid) overnight at room temperature.

\section{Tubegel digestion and sample clean up}

For each sample, $30 \mu \mathrm{g}$ of proteins were used for downstream proteomics analyses. The samples were polymerized in a $10 \%$ polyacrylamide gel containing $4 \%$ SDS and subsequently fixed with a fixing reagent ( $50 \%$ methanol, $12 \%$ acetic acid) for $30 \mathrm{~min}$ at room temperature. The gel was cut into small pieces (1 $\mathrm{mm}^{3}$ ) before being washed three times with $50 \mathrm{mM}$ TEAB/50\% acetonitrile ( $\left.\mathrm{v} / \mathrm{v}\right)$ and dehydrated with $100 \%$ acetonitrile. Next, samples were reduced using $5 \mathrm{mM}$ Tris(2-carboxyethyl) phosphine (TCEP) at 57 ${ }^{\circ} \mathrm{C}$ for 60 min followed by alkylation with $10 \mathrm{mM}$ methyl methanethiosulfonate (MMTS) for $60 \mathrm{~min}$ at room temperature with occasional vortexing. The gel pieces were washed in $500 \mu \mathrm{L}$ of $50 \mathrm{mM}$ TEAB, dehydrated in $500 \mu \mathrm{L}$ acetonitrile, and re-hydrated with $500 \mu \mathrm{L}$ of $50 \mathrm{mM}$ TEAB. The final dehydration step was performed using $100 \mu \mathrm{L}$ acetonitrile. Trypsinization ( $1.5 \mu \mathrm{g}$ trypsin) was performed at $37^{\circ} \mathrm{C}$ for $16 \mathrm{~h}$. The digested peptides were centrifuged at $6000 \times \mathrm{g}$ for $10 \mathrm{~min}$ to collect the supernatant and stored at $-20{ }^{\circ} \mathrm{C}$ (protocol modified from [17]. The samples were lyophilized and $30 \mu \mathrm{L}$ of the dissolution buffer (0.5 M TEAB, pH 8.5) was added to each sample.

\section{D LC-MS/MS analysis}

The separation of peptides was performed with an Eksigent nanoLC Ultra and ChiPLC-nanoflex (Eksigent, Dublin, CA, USA) in Trap-Elute configuration. The samples were desalted with a Sep-Pak tC $18 \mu \mathrm{L}$ Elution Plate (Waters, Miltford, MA, USA), and reconstituted using $20 \mathrm{~mL}$ of $2 \%$ acetonitrile and $0.05 \%$ formic acid. Five $\mathrm{mL}$ of each sample was loaded on a $200 \mu \mathrm{m} \times 0.5 \mathrm{~mm}$ trap column and eluted on a $75 \mu \mathrm{m} \times 15$ $\mathrm{cm}$ analytical column (ChromXP C18-CL, $3 \mu \mathrm{m}$ ). A gradient formed by mobile phase A ( $2 \%$ acetonitrile, $0.1 \%$ formic acid) and mobile phase B ( $98 \%$ acetonitrile, $0.1 \%$ formic acid) was used to separate the sample content at a $0.3 \mu \mathrm{L} / \mathrm{min}$ flow rate. The following gradient elution was used for peptide separation: 0 to $5 \%$ of mobile phase B in $1 \mathrm{~min}, 5$ to $12 \%$ of mobile phase B in $15 \mathrm{~min}, 12$ to $30 \%$ of mobile phase $B$ in $104 \mathrm{~min}, 30$ to $90 \%$ of mobile phase B in $2 \mathrm{~min}, 90$ to $90 \%$ in $7 \mathrm{~min}, 90$ to $5 \%$ in $3 \mathrm{~min}$ and held at $5 \%$ of mobile phase $B$ for 13 min (protocol modified from [33]. 


\section{Protein identification and quantification}

Peptide identification was carried out with the ProteinPilot 5.0 software Revision 4769 (AB SCIEX) using the Paragon database search algorithm (5.0.0.0.4767) and the integrated false discovery rate (FDR) analysis function. The data were searched against protein sequence databases downloaded from Uniport on May 2018 (total 119356 entries). The MS/MS spectra obtained were searched using the following user-defined search parameters: Sample Type: Identification; Cysteine Alkylation: MMTS; Digestion: Trypsin; Instrument: TripleTOF5600; Special Factors: None; Species: None; ID Focus: Biological Modification; Database for 2018_May_uniprot-zebrafish.fasta; Search Effort: Thorough; FDR Analysis: Yes. The MS/MS spectra were searched against a decoy database to estimate FDR for peptide identification. The decoy database consisted of reversed protein sequences from the Uniprot zebrafish database. FDR analysis was performed on the dataset and peptides identified with a confidence interval $\geq 95 \%$ were taken into account.

\section{KEGG and Gene ontology (GO) functional pathways analysis}

To analyse functional pathways associated to protein identified from deyolked and non-deyolked samples, KEGG analysis was performed. The FASTA files were submitted to online server "KAAS - KEGG Automatic Annotation Server" (https://www.genome.jp/kegg/kaas/) in order to get KEGG Orthology (KO) assignments [34]. To map KEGG pathways, the obtained KO numbers were submitted to KEGG mapper web server (http://www.genome.jp/kegg/tool/map_pathway2.html) [35].

GO annotation results and pathway of differentially expressed proteins in pairwise comparisons were obtained using Panther (Panther14.0, 2018_04) ([36]. The web conversion tool (https://biodbnetabcc.ncifcrf.gov) was used to convert unmapped UniProt Accession IDs to ZFIN ID. The web tool Biomart was used to convert unmapped ZFIN IDs to Gene stable ID and to manually identify the unmapped IDs by gene names [37]. Uniprot was used to identify protein IDs discontinued (deleted) in the 2018_11 release [38].

\section{Results}

\section{Efficiency of the new extraction protocol}


We compared our protocols to the protocols by Link et al. [16], which were based on one-step deyolking with or without subsequent washing steps. For a fair comparison, we compared the reference one-step deyolking procedure without washing [16] to our one-step deyolking procedure without washing, and the reference one-step deyolking plus double wash procedure [16] to our three-step deyolking plus single wash procedure.

Both methods resulted in a reduction of yolk proteins, and the washing steps further depleted the protein content. Nevertheless, our new protocol yielded a larger number of unique proteins from a smaller number of embryos. We obtained approximately 1.7 -fold increase in protein concentration per embryo sample when applying the one-step deyolking process. When using our three-step deyolking + single wash protocol, the protein yields per embryo sample were 3.1- and 2.5-fold higher (1-cell and high stage embryos, respectively) than those obtained with the reference protocols [16] with one-step deyolking + double wash (Fig. 1A). The effective number of 1-cell stage embryos needed to collect a workable amount of protein $(30 \mu \mathrm{g})$ was approximately 2 or 3 times lower when using our one-step deyolking or three-step deyolking + single wash protocols, respectively; for the high stage embryos, this number of embryos was approximately 2 times lower than that of the respective reference protocols (Fig. 1B). Also, compared to our new protocol, less amount of proteins was harvested with the reference protocol (Fig. 1B).

\section{Proteome in deyolked versus non-deyolked samples}

Generally, the amount of extracted total protein per embryo increased with the developmental advancement of the embryo, and the deyolking procedure greatly reduced the protein concentration. However, this reduction was decreasing from over 25 -fold in cleavage stages (1-cell, 16-cell and 32-cell stages) to approximately 15 -fold in the bud stage (Table 1 ).

Analysis of the digested protein samples using the 1D MS/MS shotgun proteomics approach (1D shotgun) consistently demonstrated that the deyolking procedure resulted in a greater number of unique proteins being identified (Supplementary file 1). In the non-deyolked samples, the total numbers of proteins identified throughout the developmental stages were relatively consistent, ranging from 338 to 434 proteins identified in the 1-cell and bud stages, respectively. By comparison, the numbers of proteins identified in deyolked samples in all the developmental stages were considerably higher than in the nondeyolked counterparts. In the cleavage stages, these differences were approximately two-fold, and increased to over three-fold in the later developmental stages, ranging from 696 to 1687 proteins identified in the 1-cell and bud stages, respectively (Fig. 2 A, Supplementary file 1). In contrast to the nondeyolked samples, where there was no apparent correlation between the developmental progression and the total number of proteins identified, deyolked samples resulted in a consistent number of proteins identified throughout cleavage stages (1-cell, 16-cell, and 32-cell), which considerably increased in the 
later developmental stages (Fig. 2A). Most of the proteins identified in the non-deyolked samples were also found in the deyolked counterparts (Fig. 2A, Supplementary file 1). The number of proteins unique to the non-deyolked samples (that is, not found in the deyolked counterparts) was relatively stable throughout the developmental stages. In contrast, most of the proteins identified in the deyolked samples were unique, meaning that they were not found in the non-deyolked counterparts, and the number of unique proteins apparently increased throughout the embryonic development from the cleavage stages to the bud stage (Fig. 2A, Supplementary file 1).

Among the 504 proteins unique to non-deyolked samples, most of them were specific to the cleavage stages, and 42 proteins were found in all the developmental stages. By comparison, out of 2129 proteins unique to the deyolked samples, 420 proteins were found in the cleavage stages only, 266 proteins were found commonly in all the deyolked samples. In contrast to the non-deyolked sample counterparts, a substantial proportion of unique proteins was found in either or both oblong and bud stages. In total, 465 proteins were present in both non-deyolked and deyolked samples across all the developmental stages (Fig. 2B, Supplementary file 1).

\section{Functional annotations of the proteome}

In both non-deyolked and deyolked samples, the identified proteins were substantially involved in metabolic, ribosome, and biosynthesis of secondary metabolite and proteasome pathways, while enrichments specific to sampling protocol and/or developmental stage were found in certain pathways, such as in proteasome, RNA transport or thermogenesis pathways (Table 2).

Analysis of representation of the identified proteins annotated to functional Gene Ontology (GO) terms revealed multiple processes, functions and components overrepresented and underrepresented in both non-deyolked and deyolked samples, with some of them specific to the developmental stage (Fig. 3 and Supplementary File 2).

To distinguish the effect of the extraction protocol (non-deyolked versus deyolked samples) from the biological features (natural representation of proteins at given developmental stage), we used functional annotations of proteins represented in both non-deyolked and deyolked samples from all the developmental stages as a filtering criterion. In this way, shared GO terms were established by: the same proteins identified in samples from both extraction methods ("Shared" dataset); different proteins in both datasets ("unique NDY" and "unique DY" datasets) enriching the same terms; or partially the same and partially different proteins ("Shared" and "unique NDY", "Shared" and "unique DY", and all the three datasets). Whereas, unique GO terms were established exclusively by proteins from either "unique NDY" or "unique DY" datasets. 
Clearly, the deyolking procedure yielded a considerable number of unique GO terms, which were not annotated with the proteins identified in the non-deyolked samples (Supplementary File S3). The most prominent, developmentally relevant examples included enrichment in: cellular component organization, RNA splicing, DNA replication, intracellular transport, cell cycle, translational initiation, and mitochondrial organization, transport and gene expression (biological process); ATP binding, GTP binding, NADH dehydrogenase activity, ribonucleoprotein complex binding, translation initiation factor activity, ribosome binding, and ligase activity (molecular function); chromosome, endoplasmic reticulum, Golgi-associated vesicle, polysome, spliceosomal complex, cytochrome, and mitochondrial ribosome, matrix and respiratory chains I and II (cellular component). Similarly, underrepresentation in unique GO terms was developmentally relevant, and it included: cell-cell signalling, chemical synaptic transmission, intracellular signal transduction, and immune response (biological process); DNA-binding transcription factor activity, transcription regulator activity, channel activity, G protein-coupled receptor activity, and kinase activity (molecular function); cell surface, extracellular region, and plasma membrane-bounded cell projection (cellular component). In contrast to abundance of unique GO terms annotated with the "unique DY" dataset, there were very few unique GO terms associated with "unique NDY" dataset, with most notably enriched terms in molecular function: carbohydrate binding and endopeptidase regulator activity (Supplementary Files S2 and S3).

\section{Discussion}

The improved deyolking procedure resulted in considerably high quantity of the extracted total protein (Fig. 1A) We have identified 1355 proteins, and 37 out of 51 (73\%) proteins identified by Link et al. [16] were found in our dataset. We used three times less embryos in our procedure (Fig. 1B) than in the reference procedure [16]. Consequently, we were able to conduct the proteomics analysis of zygotic and cleavage stages of zebrafish for the first time. Most of the proteins identified in the cleavage stages were unique to these stages of development (786 out of 1494; Fig 2B). This indicates a massive dynamics of zebrafish developmental proteome.

KEGG analysis showed that ribosome, biosynthesis of secondary metabolites, carbon metabolism and proteasome pathways were detectable in all the samples (Table 2). Also, a number of Go terms were detected in both deyolked and non-deyolked datasets (Supplementary file S3). Nevertheless, we have observed a substantial increase in the number of identified unique proteins in the deyolked samples as compared to the non-deyolked counterparts. Consequently, they enriched a number of developmentally relevant GO terms, such as the cell cycle, mitochondrial organization and functions or translation initiation, which were not enriched in the non-deyolked samples (Supplementary file S3). These functional terms are essential for the proper growth and development of early stage of embryos [25-28] Knowledge of developmentally relevant proteome will aid understanding the regulation of early embryonic development. The underrepresented GO terms in the deyolked samples were mainly related to cellular signaling, transcription, G protein-coupled receptor activity and cell surface (Supplementary file S3). These terms were not found underrepresented in the non-deyolked samples. In contrast to the significant GO terms found uniquely in the deyolked samples, there were very few unique GO terms were associated 
with "unique NDY" dataset (Supplementary file S3); this indicates that the presence of many embryonic proteins is masked due to the high abundance of yolk.

The functional annotation of cleavage stage proteome is concordant with the canonical knowledge of the catabolism, cell cycle, subcellular organization, and the transcriptional quiescence of pre-MZT embryos $[24,29]$. Moreover, our data suggest active translation-related processes in the very early embryos. Since zygotic transcripts are not produced yet [8], maternally-provided mRNAs [30] are used to produce the translational machinery and perform the translation. Quantitative proteome analysis throughout the development would be needed to determine the extent of this process, though.

Although the dechorionation/deyolking procedure generally resulted in a substantial increase in the number of identified proteins, it also resulted in a loss of certain proteins as compared to the nondeyolked counterparts (Fig. 2A), similarly to a study on $5 \mathrm{dpf}$ zebrafish larvae [21]. Most of the previous

proteomic studies did not address the problem of protein depletion due to the deyolking process, and they only used deyolked embryos for the analyses $[16,21,22]$. In the present study, approximately $30 \%$ of the proteins at cleavage stages and $12 \%$ at oblong and bud stages were not identified after the deyolking (Fig. 2, Supplementary File 1). The GO analysis revealed that these lost proteins are involved in a number of biological processes (translation, protein folding and mitochondrial organization), molecular functions ( generation of precursor metabolites and energy) and cellular component (ribosome and cytosol;

Supplementary File 2). Therefore, our results suggest that deyolked and non-deyolked samples should be analyzed in parallel to extract a reliable information on the proteome in embryonic development.

\section{Conclusions}

We have established an effective deyolking procedure for the proteome analysis of the early stages of zebrafish embryos. Elimination of most of the yolk from early stages of embryos significantly enhanced the identification of cellular proteins with LC-MS-based shotgun proteomics analysis. The improved protocol is applicable to low-input material, enabling investigation of the earliest stages of development. Also, we demonstrate that the deyolking procedure results in the depletion of certain parts of the proteome that can be important in embryonic development. Thus, we suggest that both deyolked and non-deyolked samples should be processed in parallel to ensure a reliable coverage of the proteome during the embryogenesis. Our deyolking procedure will improve both qualitative and quantitative proteome analyses throughout embryonic development of polylecithal animals, such as fish, amphibians, reptiles, and birds.

\section{Abbreviations}

ND: non-deyolk DY: deyolk SH: shared iTRAQ: relative and absolute quantification LC-MS/MS: liquid chromatography-tandem mass spectrometry 1D LC-MS/MS: one dimension liquid chromatographytandem mass spectrometry MZT: maternal-to-zygotic transition PBS: phosphate buffered saline SDS: sodium dodecyl sulfate TCEP: tris-(2-carboxyethyl) phosphine MMTS: methyl methane-thiosulfonate 
TEAB: triethylammonium bicarbonate ACN: acetonitrile FDR: false discovery rate COG: clusters of orthologous groups GO: gene ontology KEGG: kyoto encyclopedia of genes and genomes

\section{Declarations}

\section{Ethics approval and consent to participate}

No human subjects were involved in this study. For the procedures used and material collected, no formal ethics approval is needed. All experimental procedures and animal handling complied with the guidelines of Norwegian regulation for laboratory animal experimentation (The Norwegian Animal Protection Act, No. 73 of 20 December 1974, Section 20-22, amended 19 June 2009).

\section{Consent to publish}

Not applicable

\section{Availability of data and materials}

All the data are available in Supplementary Files. Any other information is available upon request.

\section{Competing interests}

The authors declare no competing interests.

\section{Funding}

The research was supported by the InnContro/ project (Research Council of Norway, grant \#275786, granted to IB), Nord University (scholarship for PK), and Protein and Proteomics Centre, Department of Biological Sciences, National University of Singapore (granted to LQ). The funding bodies had no role in the design of the study and collection, analysis, and interpretation of data and in writing the manuscript.

\section{Author contributions}

PK: performed sampling, laboratory work, data analysis and drafted the manuscript. CP: sampling and drafted the manuscript. PPD: laboratory work and drafted the manuscript. LTK: laboratory work. IB, LQ 
and SDJ: designed and coordinated the study, performed data analyses, and drafted the manuscript. All authors approved the final manuscript.

\section{Acknowledgments}

We would like to thank Felix Müller, Research fellow at Faculty of Biosciences and Aquaculture, Nord University, who helped in embryo collections. PK wishes to thank Nord University for funding the PhD scholarship and travel funding.

\section{References}

1. Amsterdam A, Hopkins $\mathrm{N}$ : Mutagenesis strategies in zebrafish for identifying genes involved in development and disease. Trends in genetics 2006, 22(9):473-478.

2. Brownlie A, Donovan A, Pratt SJ, Paw BH, Oates AC, Brugnara C, Witkowska HE, Sassa S, Zon LI: Positional cloning of the zebrafish sauternes gene: a model for congenital sideroblastic anaemia. Nature genetics 1998, 20(3):244.

3. Grosser T, Yusuff S, Cheskis E, Pack MA, FitzGerald GA: Developmental expression of functional cyclooxygenases in zebrafish. Proceedings of the National Academy of Sciences 2002, 99(12):84188423.

4. Gündel U, Benndorf $D$, von Bergen $M$, Altenburger R, Küster E: Vitellogenin cleavage products as indicators for toxic stress in zebra fish embryos: a proteomic approach. Proteomics 2007, 7(24):4541-4554.

5. Hanisch K, Küster E, Altenburger R, Gündel U: Proteomic signatures of the zebrafish (Danio rerio) embryo: sensitivity and specificity in toxicity assessment of chemicals. International journal of proteomics 2010, 2010.

6. Howe K, Clark MD, Torroja CF, Torrance J, Berthelot C, Muffato M, Collins JE, Humphray S, McLaren K, Matthews $L$ : The zebrafish reference genome sequence and its relationship to the human genome. Nature 2013, 496(7446):498.

7. Aanes H, Winata CL, Lin CH, Chen JP, Srinivasan KG, Lee SG, Lim AY, Hajan HS, Collas P, Bourque G: Zebrafish mRNA sequencing deciphers novelties in transcriptome dynamics during maternal to zygotic transition. Genome research 2011, 21(8):1328-1338.

8. Heyn P, Kircher M, Dahl A, Kelso J, Tomancak P, Kalinka AT, Neugebauer KM: The earliest transcribed zygotic genes are short, newly evolved, and different across species. Cell reports 2014, 6(2):285-292.

9. Mehjabin R, Xiong L, Huang R, Yang C, Chen G, He L, Liao L, Zhu Z, Wang Y: Full-Length Transcriptome Sequencing and the Discovery of New Transcripts in the Unfertilized Eggs of Zebrafish (Danio rerio). G3: Genes, Genomes, Genetics 2019:g3. 200997.202019.

10. Nudelman G, Frasca A, Kent B, Sadler KC, Sealfon SC, Walsh MJ, Zaslavsky E: High resolution annotation of zebrafish transcriptome using long-read sequencing. Genome research 2018, 
28(9):1415-1425.

11. White RJ, Collins JE, Sealy IM, Wali N, Dooley CM, Digby Z, Stemple DL, Murphy DN, Billis K, Hourlier $\mathrm{T}$ : A high-resolution mRNA expression time course of embryonic development in zebrafish. Elife 2017, 6:e30860.

12. De Souza AG, MacCormack TJ, Wang N, Li L, Goss GG: Large-scale proteome profile of the zebrafish (Danio rerio) gill for physiological and biomarker discovery studies. Zebrafish 2009, 6(3):229-238.

13. Groh KJ, Nesatyy VJ, Segner H, Eggen RI, Suter MJ-F: Global proteomics analysis of testis and ovary in adult zebrafish (Danio rerio). Fish physiology and biochemistry 2011, 37(3):619-647.

14. Singh SK, Sundaram CS, Shanbhag S, Idris MM: Proteomic profile of zebrafish brain based on twodimensional gel electrophoresis matrix-assisted laser desorption/ionization MS/MS analysis. Zebrafish 2010, 7(2):169-177.

15. Zhang J, Lanham KA, Peterson RE, Heideman W, Li L: Characterization of the adult zebrafish cardiac proteome using online pH gradient strong cation exchange-RP 2D LC coupled with ESI MS/MS. Journal of separation science 2010, 33(10):1462-1471.

16. Link V, Shevchenko A, Heisenberg C-P: Proteomics of early zebrafish embryos. BMC developmental biology 2006, 6(1):1.

17. Lu W, Yin X, Liu X, Yan G, Yang P: Response of peptide intensity to concentration in ESI-MS-based proteome. Science China Chemistry 2014, 57(5):686-694.

18. Vaidyanathan S, Kell DB, Goodacre R: Selective detection of proteins in mixtures using electrospray ionization mass spectrometry: influence of instrumental settings and implications for proteomics. Analytical chemistry 2004, 76(17):5024-5032.

19. Lucitt MB, Price TS, Pizarro A, Wu W, Yocum AK, Seiler C, Pack MA, Blair IA, FitzGerald GA, Grosser T: Analysis of the zebrafish proteome during embryonic development. Molecular \& Cellular Proteomics 2008, 7(5):981-994.

20. Rahlouni F, Szarka S, Shulaev V, Prokai L: A Survey of the Impact of Deyolking on Biological Processes Covered by Shotgun Proteomic Analyses of Zebrafish Embryos. Zebrafish 2015, 12(6):398-407.

21. Tay TL, Lin Q, Seow TK, Tan KH, Hew CL, Gong Z: Proteomic analysis of protein profiles during early development of the zebrafish, Danio rerio. Proteomics 2006, 6(10):3176-3188.

22. Alli Shaik A, Wee S, Li RHX, Li Z, Carney TJ, Mathavan S, Gunaratne J: Functional mapping of the zebrafish early embryo proteome and transcriptome. Journal of proteome research 2014, 13(12):5536-5550.

23. Lößner C, Wee S, Ler SG, Li RH, Carney T, Blackstock W, Gunaratne J: Expanding the zebrafish embryo proteome using multiple fractionation approaches and tandem mass spectrometry. Proteomics 2012, 12(11):1879-1882.

24. Tadros W, Lipshitz HD: The maternal-to-zygotic transition: a play in two acts. Development 2009, 136(18):3033-3042. 
25. Gong Y, Mo C, Fraser SE: Planar cell polarity signalling controls cell division orientation during zebrafish gastrulation. Nature 2004, 430(7000):689.

26. Sugiyama M, Sakaue-Sawano A, limura T, Fukami K, Kitaguchi T, Kawakami K, Okamoto H, Higashijima S-i, Miyawaki A: Illuminating cell-cycle progression in the developing zebrafish embryo. Proceedings of the National Academy of Sciences 2009, 106(49):20812-20817.

27. Artuso L, Romano A, Verri T, Domenichini A, Argenton F, Santorelli FM, Petruzzella V: Mitochondrial DNA metabolism in early development of zebrafish (Danio rerio). Biochimica et Biophysica Acta (BBA)-Bioenergetics 2012, 1817(7):1002-1011.

28. Sun J, Yan L, Shen W, Meng A: Maternal Ybx1 safeguards zebrafish oocyte maturation and maternalto-zygotic transition by repressing global translation. Development 2018, 145(19): $\operatorname{dev} 166587$.

29. Lee MT, Bonneau AR, Giraldez AJ: Zygotic genome activation during the maternal-to-zygotic transition. Annual review of cell and developmental biology 2014, 30:581-613.

30. Lubzens E, Bobe J, Young G, Sullivan CV: Maternal investment in fish oocytes and eggs: the molecular cargo and its contributions to fertility and early development. Aquaculture 2017, 472:107143.

31. Westerfield M: A guide for the laboratory use of zebrafish (Danio rerio). The zebrafish book 2000, 4.

32. Kimmel CB, Ballard WW, Kimmel SR, Ullmann B, Schilling TF: Stages of embryonic development of the zebrafish. Developmental dynamics 1995, 203(3):253-310.

33. Suriyanarayanan T, Qingsong L, Kwang LT, Mun LY, Seneviratne CJ: Quantitative proteomics of strong and weak biofilm formers of Enterococcus faecalis reveals novel regulators of biofilm formation. Molecular \& Cellular Proteomics 2018, 17(4):643-654.

34. Moriya Y, Itoh M, Okuda S, Yoshizawa AC, Kanehisa M: KAAS: an automatic genome annotation and pathway reconstruction server. Nucleic acids research 2007, 35(suppl_2):W182-W185.

35. Kanehisa M, Sato $Y$, Kawashima M, Furumichi M, Tanabe M: KEGG as a reference resource for gene and protein annotation. Nucleic acids research 2015, 44(D1):D457-D462.

36. Mi H, Huang X, Muruganujan A, Tang H, Mills C, Kang D, Thomas PD: PANTHER version 11: expanded annotation data from Gene Ontology and Reactome pathways, and data analysis tool enhancements. Nucleic acids research 2016, 45(D1):D183-D189.

37. Zerbino DR, Achuthan P, Akanni W, Amode MR, Barrell D, Bhai J, Billis K, Cummins C, Gall A, Girón CGJNar: Ensembl 2018. 2017, 46(D1):D754-D761.

38. Apweiler R, Bairoch A, Wu CH, Barker WC, Boeckmann B, Ferro S, Gasteiger E, Huang H, Lopez R, Magrane M: UniProt: the universal protein knowledgebase. Nucleic acids research 2004, 32(suppl_1):D115-D119.

\section{Tables}


Table 1 - The amount of protein extracted from deyolked versus non-deyolked embryos.

\begin{tabular}{lrrrrr}
\hline & Number of embryos & Total sample volume & \multicolumn{2}{c}{$\begin{array}{c}\text { Amount of extracted protein } \\
(\mu \mathrm{g})\end{array}$} \\
Developmental stage & & $\mu \mathrm{L}$ & Total & per $\mu \mathrm{L}$ & per embryo \\
\hline Non- deyolked & & & & & \\
1-cell & 28 & 119 & 395.08 & 3.32 & 14.11 \\
\hline 16-cell & 20 & 88 & 327.36 & 3.72 & 16.37 \\
\hline 32-cell & 40 & 170 & 697.00 & 4.10 & 17.42 \\
\hline Oblong & 20 & 120 & 478.80 & 3.99 & 23.94 \\
\hline Bud & 20 & 92 & 524.40 & 5.70 & 26.22 \\
\hline Deyolked & & & & & \\
1-cell & 575 & 94 & 304.56 & 3.24 & 0.53 \\
\hline 16-cell & 300 & 58 & 191.41 & 3.30 & 0.63 \\
\hline 32-cell & 400 & 99 & 277.22 & 2.81 & 0.69 \\
\hline Oblong & 225 & 42 & 246.54 & 5.87 & 1.09 \\
\hline Bud & 250 & 70 & 413.70 & 5.91 & 1.65 \\
\hline
\end{tabular}

Table 2 - Significant $(p<0.05)$ pathways identified by KEGG analysis of proteins from non-deyolked (NDY) cleavage, oblong \& bud stage zebrafish embryos, and their deyolked (DY) counterparts. Numbers of proteins mapped to annotated pathways are given. 


\begin{tabular}{|c|c|c|c|c|c|c|c|}
\hline Pathway Name & $\begin{array}{l}\text { NDY- } \\
\text { Cleavage } \\
\text { Stage- } \\
\text { Unique } \\
\end{array}$ & $\begin{array}{l}\text { NDY- } \\
\text { Oblong \& } \\
\text { Bud stages } \\
\text { Unique } \\
\end{array}$ & $\begin{array}{l}\text { NDY- } \\
\text { Common } \\
\text { in all } \\
\text { stages }\end{array}$ & $\begin{array}{l}\text { DY- } \\
\text { Cleavage } \\
\text { stage } \\
\text { Unique } \\
\end{array}$ & $\begin{array}{l}\text { DY-Oblong } \\
\text { \& Bud } \\
\text { stages } \\
\text { Unique }\end{array}$ & $\begin{array}{l}\text { DY- } \\
\text { Common } \\
\text { in all } \\
\text { stages } \\
\end{array}$ & $\begin{array}{l}\text { Shared } \\
\text { proteins }\end{array}$ \\
\hline $\begin{array}{l}\text { map01100 Metabolic } \\
\text { pathways }\end{array}$ & 22 & 23 & 6 & 66 & 62 & 87 & 69 \\
\hline map03010 Ribosome & 27 & 11 & 2 & 21 & 19 & 11 & 52 \\
\hline $\begin{array}{l}\text { map01110 } \\
\text { Biosynthesis of } \\
\text { secondary } \\
\text { metabolites }\end{array}$ & 12 & 11 & 3 & 21 & 25 & 27 & 32 \\
\hline $\begin{array}{l}\text { map04714 } \\
\text { Thermogenesis }\end{array}$ & 2 & 6 & 1 & 28 & 6 & 37 & 20 \\
\hline $\begin{array}{l}\text { map01200 Carbon } \\
\text { metabolism }\end{array}$ & 6 & 6 & 1 & 10 & 12 & 21 & 23 \\
\hline $\begin{array}{l}\text { map04141 Protein } \\
\text { processing in } \\
\text { endoplasmic } \\
\text { reticulum }\end{array}$ & 4 & 0 & 0 & 5 & 14 & 11 & 20 \\
\hline $\begin{array}{l}\text { map03050 } \\
\text { Proteasome }\end{array}$ & 14 & 4 & 1 & 1 & 15 & 0 & 14 \\
\hline $\begin{array}{l}\text { map00010 Glycolysis } \\
\text { / Gluconeogenesis }\end{array}$ & 6 & 3 & 0 & 2 & 6 & 6 & 12 \\
\hline $\begin{array}{l}\text { map00071 Fatty acid } \\
\text { degradation }\end{array}$ & 3 & 1 & 0 & 9 & 1 & 11 & 9 \\
\hline $\begin{array}{l}\text { map01212 Fatty acid } \\
\text { metabolism }\end{array}$ & 8 & 3 & 0 & 7 & 0 & 10 & 8 \\
\hline $\begin{array}{l}\text { map04530 Tight } \\
\text { junction }\end{array}$ & 5 & 5 & 2 & 6 & 13 & 2 & 7 \\
\hline $\begin{array}{l}\text { map03013 RNA } \\
\text { transport }\end{array}$ & 7 & 0 & 0 & 3 & 22 & 1 & 6 \\
\hline map04110 Cell cycle & 9 & 2 & 0 & 3 & 12 & 1 & 5 \\
\hline $\begin{array}{l}\text { map0 } 4810 \\
\text { Regulation of actin } \\
\text { cytoskeleton }\end{array}$ & 5 & 1 & 1 & 6 & 12 & 1 & 4 \\
\hline $\begin{array}{l}\text { map04144 } \\
\text { Endocytosis }\end{array}$ & 3 & 0 & 0 & 3 & 10 & 6 & 4 \\
\hline $\begin{array}{l}\text { map00230 Purine } \\
\text { metabolism }\end{array}$ & 5 & 5 & 1 & 2 & 11 & 1 & 4 \\
\hline $\begin{array}{l}\text { map03018 RNA } \\
\text { degradation }\end{array}$ & 3 & 0 & 0 & 0 & 8 & 1 & 4 \\
\hline map04210 Apoptosis & 0 & 4 & 1 & 4 & 5 & 1 & 4 \\
\hline $\begin{array}{l}\text { map00970 } \\
\text { Aminoacyl-tRNA } \\
\text { biosynthesis }\end{array}$ & 2 & 1 & 1 & 8 & 12 & 1 & 3 \\
\hline $\begin{array}{l}\text { map03030 DNA } \\
\text { replication }\end{array}$ & 4 & 3 & 0 & 0 & 14 & 0 & 3 \\
\hline
\end{tabular}




\section{Figures}

A)
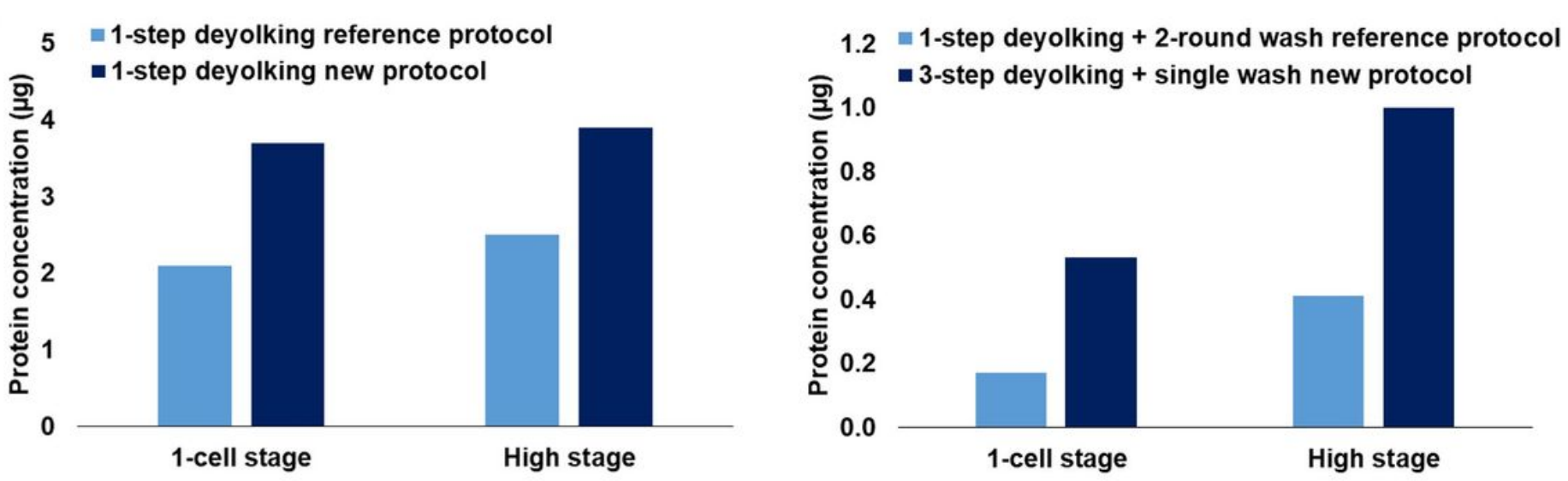

B)

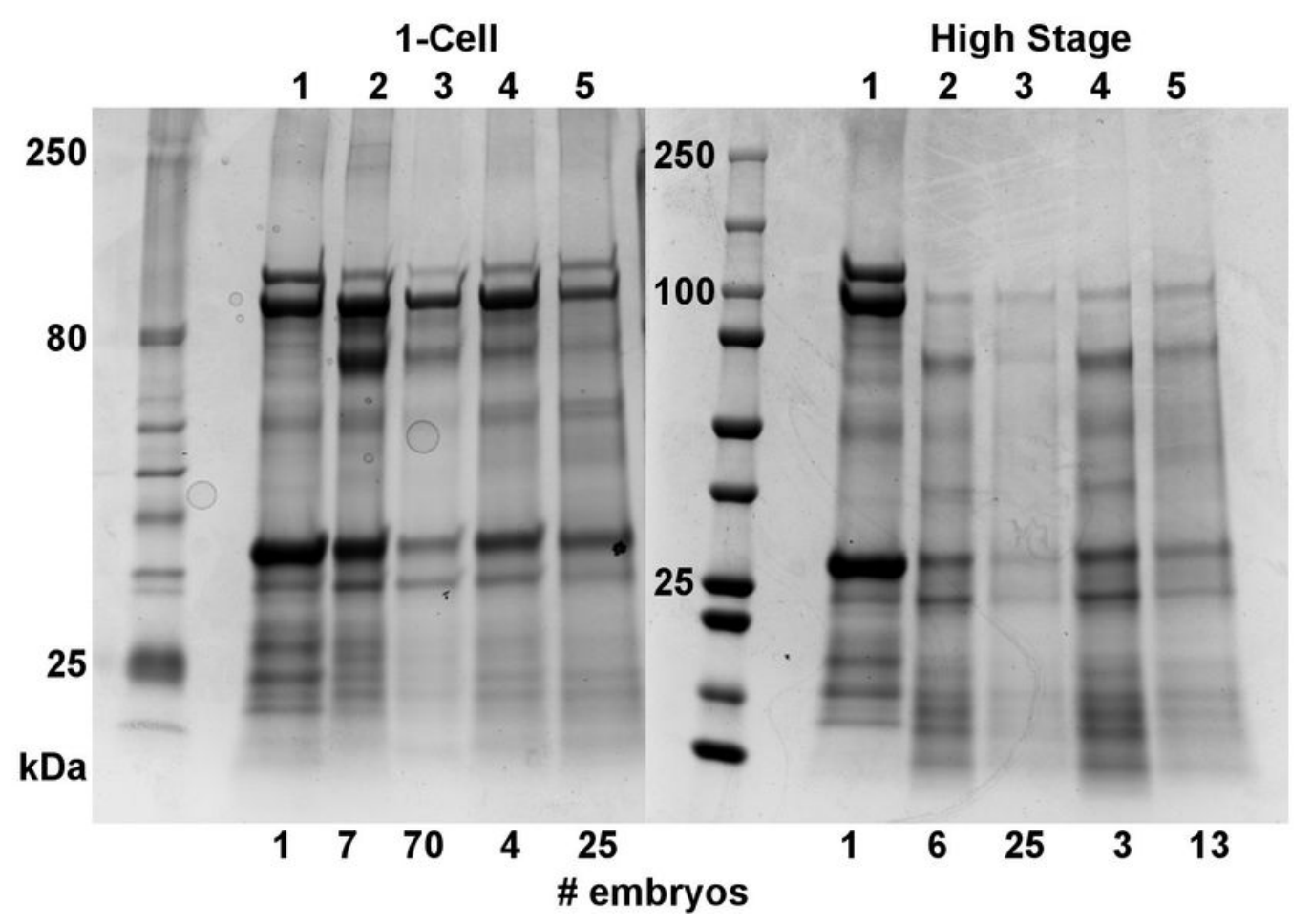

Figure 1

Comparison of the efficiencies of deyolking protocols: reference [16] versus the new one. A), Protein concentration obtained using protocols in 1-step versions (left chart) and in full versions (right chart). B), SDS-PAGE of proteins extracted from zebrafish embryos at 1-cell stage (left panel) and high stage (right panel) using the reference protocols versus new protocols. Lane 1 - non-deyolked embryo (control); Lane 2 - one-step deyolking reference protocol [16]; Lane 3 - one-step deyolking + double wash reference protocol [16]; Lane 4 - one step deyolking (new method); and Lane 5 - three-step deyolking + single wash (new method). At the bottom line, number of embryos is given for each sample, from which the proteins were extracted. 


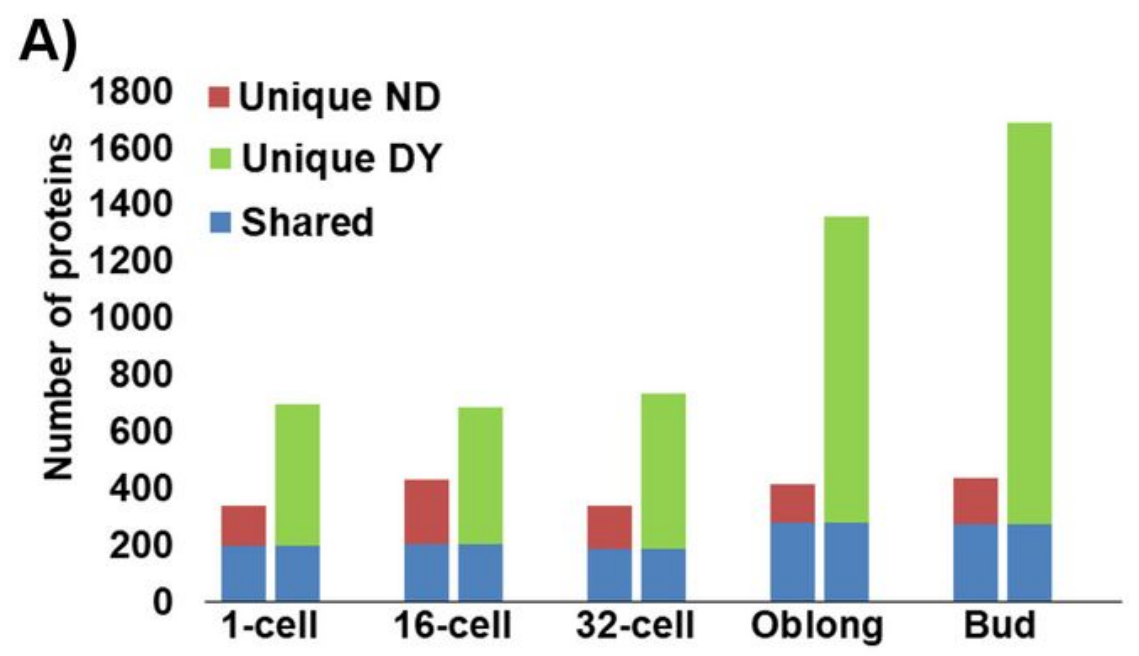

B)

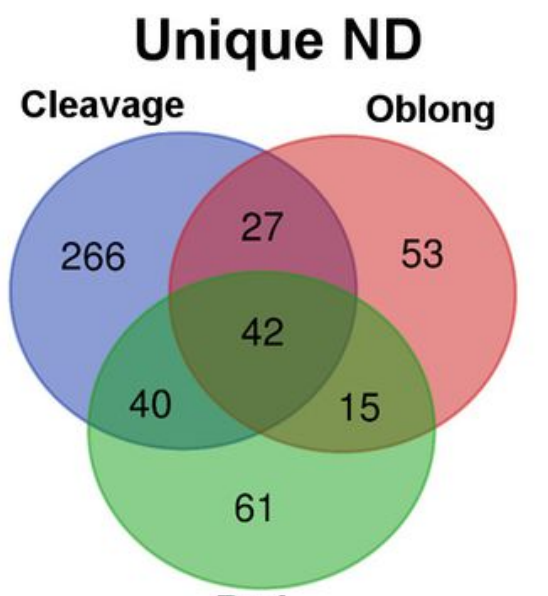

Bud
Unique DY

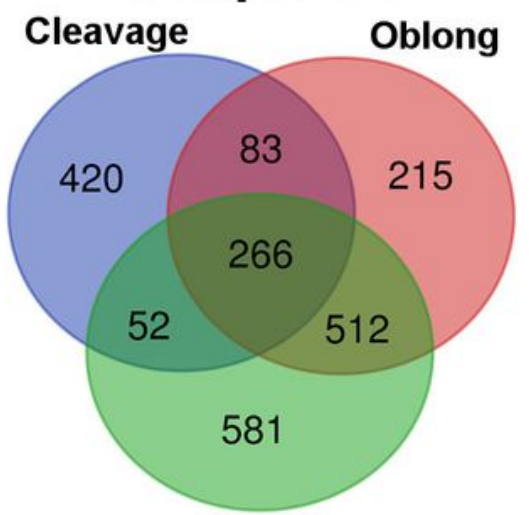

Bud

Shared

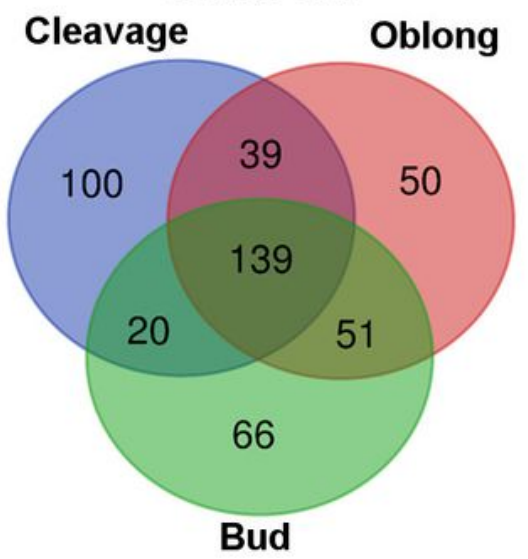

Figure 2

Numbers of proteins identified in samples from intact (non-deyolked, ND) versus deyolked (DY) zebrafish embryos. Unique proteins were found in either ND or DY embryos, whereas shared proteins were found in both ND and DY embryos. (A), total number of unique and shared proteins in ND (left column) and DE embryos (right column) at 1-cell, 16-cell, 32-cell, oblong, and bud developmental stages. (B), Specificity and overlap of the identified proteins across the critical stages of early embryonic development: cleavage 
stages (1-, 16-, and 32-cell stages combined), maternal-zygotic transition (oblong), and post-maternalzygotic transition (bud).

Non-deyolked

Shared

Deyolked

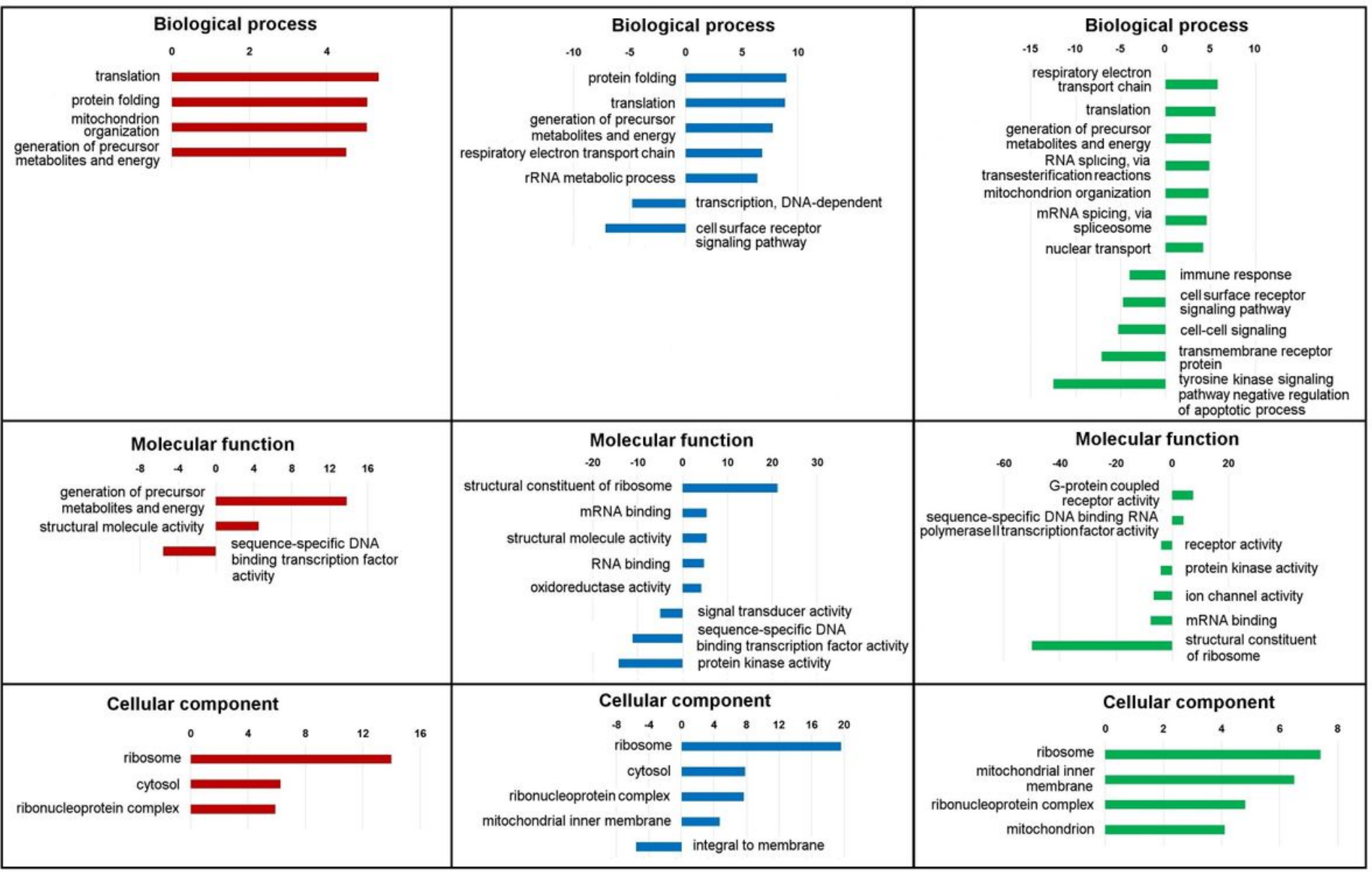

\section{Figure 3}

Significantly (FDR < 0.05) enriched Gene Ontology (GO) terms from SLIM analysis for unique deyolked, unique non-deyolked, and shared proteins grouped by biological process, molecular function, and cellular component. Representation of GO terms containing a minimum 100 reference genes and a fold change $\geq$ 4 or $\leq 4$ is given.

\section{1-Cell}

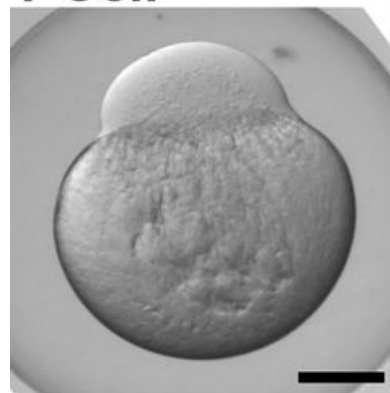

16-Cell

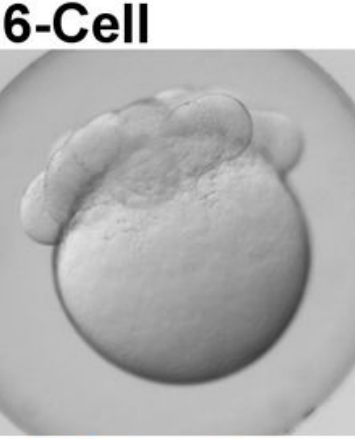

Cleavage
32-Cell

Oblong

Bud
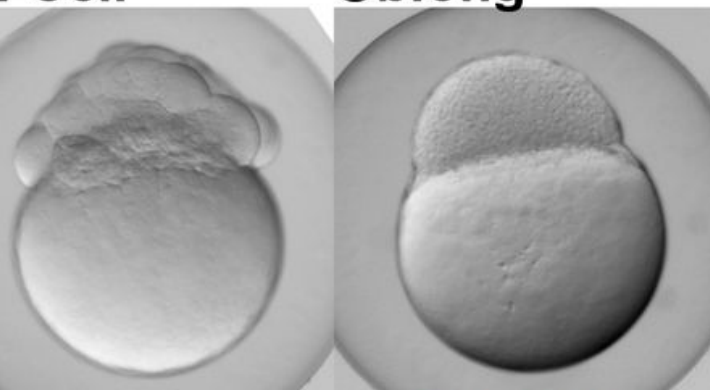

Blastula

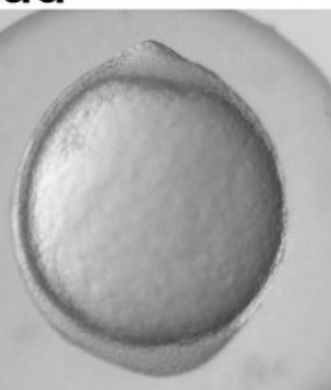

Gastrula 
Figure 4

Developmental stages of zebrafish embryos sampled in the present study. $\mathrm{Hpf}=$ hours post-fertilization at $28.5^{\circ} \mathrm{C}$.

\section{Supplementary Files}

This is a list of supplementary files associated with this preprint. Click to download.

- Supplementaryfile2.xlsx

- SupplementaryFile3.xlsx

- Supplementaryfile1.xlsx 